\title{
ANALYSIS OF DYNAMIC CAPABILITIES FOR SPECTRUM SHARING IN THE CITIZENS BROADBAND RADIO SERVICE
}

\author{
Seppo Yrjölä (Nokia, Oulu, Finland; seppo.yrjola@nokia.com); Marja Matinmikko \\ (University of Oulu, Oulu, Finland; marja.matinmikko@ee.oulu.fi); Miia Mustonen \\ (VTT Technical Research Centre of Finland, Oulu, Finland; miia.mustonen@vtt.fi); Petri \\ Ahokangas (Oulu Business School, Oulu, Finland; petri.ahokangas@oulu.fi)
}

\begin{abstract}
This paper seeks to identify and analyze the sources of value creation and capture by key stakeholders in the new Citizens Broadband Radio Service (CBRS) three tier spectrum sharing framework introduced by the US President's Council of Advanced Science \& Technology. More flexible and dynamic use of the $3.5 \mathrm{GHz}$ spectrum aims to increase the efficiency of spectrum use in delivering fast growing and converging mobile broadband and media services while paving the way to innovations in technology and business models. In this paper, we focus on key stakeholders' capability to deal with combined internal and external resources and capabilities in doing business, referred to as Dynamic Capability. Spectrum sharing, introducing a rapid change in the technology and business environments, requires dynamic capabilities from spectrum offering, spectrum utilization, and spectrum management perspectives. We focus on defining key CBRS functional domains and identifying their key antecedents, elements, and outcomes. The analysis highlights the key role of the regulator in creating a sharing framework with incentives for all the key stakeholders, with different operational and business requirements, and enabling scaling ecosystem. Increased system dynamics in spectrum sharing will introduce a need for big data analytics, near real time network management capabilities and low cost third tier general authorized access radios, leveraging dominant technology ecosystems. This study provides viewpoints for stakeholders about additional ingredients and actions, which may be relevant to promote spectrum sharing in the form of the CBRS. The concept of dynamic capabilities was found useful to analyze the sources of competitive advantage regarding CBRS spectrum sharing.
\end{abstract}

\section{INTRODUCTION}

The rapid growth in the number of mobile and wireless communication systems' users with a large range of diverse services, applications and devices [1] will require significantly more spectrum and wider continuous bandwidth than currently available [2] despite advances in spectral efficiency and network densification. In order to meet these additional spectrum demands, besides identifying more dedicated spectrum, the regulators have globally shown growing interest in novel regulatory approaches related to spectrum allocation, utilization, and management. The US President's Council of Advanced Science \& Technology (PCAST) report [3] proposed the three tier model, and underlined the role of spectrum sharing and dynamic spectrum access to find a balance between different systems and services with their different spectrum requirements and system dynamics to meet the growing spectrum crisis. Furthermore, the Federal Communications Commission (FCC) sees the opening of the $3.5 \mathrm{GHz}$ Band as " $a$ new chapter in the history of the administration of one of our nation's most precious resources-the electromagnetic radio spectrum" [4].

Early cooperation across government, industry and academia is required for any feasible and attractive spectrum sharing framework that allows several radio systems to operate in the same spectrum. Collaboration in the technology and innovation domain enables the creation and validation of the technical enablers and new system concepts, while ensuring economies of scale and scope in deployment. On one hand, the harmonized spectrum regulation has enabled multibillion business ecosystems emerge around two distinct spectrum access approaches: the mobile broadband businesses via exclusive Quality of Service (QoS) spectrum usage rights, and the unlicensed Wi-Fi ecosystem drawing from the public spurring innovations. On the other hand, to date only a subset of the spectrum sharing concepts has reached the regulation domain. Furthermore, several spectrum sharing concepts widely researched, standardized and supported by national regulatory authorities (NRA) have not scaled up as expected, TV White Space (TVWS) from US [5] as the latest example.

Based on this profound spectrum sharing work, two novel licensing based sharing models have recently emerged, the Licensed Shared Access (LSA) [6] from Europe and the three tier Citizens Broadband Radio Service (CBRS) governed by the Spectrum Access System (SAS) from the US 
[4]. For these prominent spectrum sharing concepts currently under regulatory discussion and early stage standardization, and particularly for the CBRS, there is not much prior work available in the field of strategic management and business modeling. An initial evaluation of the general spectrum sharing concept from the business modeling point of view can be found in [7] and the LSA focused analysis, e.g., from [8] and [9]. Spectrum sharing transformation has the potential to shift the market towards an open structure, enabling many new entrants and a wide range of service applications [7]. The impact of new actor introduction in terms of novel business models has been discussed by Ballon \& Dalaere [10]. The viability of the cognitive radio and spectrum sharing enabled spectrum trading market has been studied in [11]. This paper extends that work by focusing on the recent dynamic CBRS sharing concept and analyzing the sources of competitive advantage, value creation and capture using the Dynamic Capabilities (DC) approach. Teece et al. [12] introduced the concept of DC to refer to capability to deal with combined internal and external resources and capabilities in doing business in environments of rapid technological change. Teece defines DC as "the firm's ability to integrate, build and reconfigure internal and external competences to address rapidly changing environments" [12]. DC can be described in terms of which actions are taken to adjust a company`s resources into innovate forms of competitive advantage. By analyzing the CBRS concept from the DC perspective, this paper seeks to answer the following research questions:

1) What are DCs required for the key domains of spectrum sharing using CBRS?

2) What are the key technology enablers needed to exploit CBRS spectrum sharing in mobile broadband networks?

3) Could this be of help for key stakeholders and regulators for implementing CBRS and SAS?

The rest of the paper is organized as follows. First, the three tier CBRS sharing framework and the SAS concept are presented and defined in section 2. Third, the theory of DCs is described including an interpretation of the CBRS concept from the DC perspective. In section 4, the analysis is further deepened into the technology enabler assessment. Finally, conclusions are drawn.

\section{CITIZENS BROADBAND RADIO SERVICE SPECTRUM SHARING FRAMEWORK}

The Presidential Memorandum in 2013 further strengthened the key policy messages of the PCAST report [13] from 2012, indicating that "...we must make available even more spectrum and create new avenues for wireless innovation. One means of doing so is by allowing and encouraging shared access to spectrum that is currently allocated exclusively for Federal use. Where technically and economically feasible, sharing can and should be used to enhance efficiency among all users and expedite commercial access to additional spectrum bands, subject to adequate interference protection for Federal users, ... We should also seek to eliminate restrictions on commercial carriers' ability to negotiate sharing arrangements with agencies. To further these efforts, while still safeguarding protected incumbent systems that are vital to Federal interests and economic growth, this memorandum directs agencies and offices to take a number of additional actions to accelerate shared access to spectrum."

After intensive discussion and consultation with the interest groups the FCC released an order on reconsideration and the second report and order to establish new rules for shared use of the 3550-3700 MHz band in May 2016 [14]. The framework defines a contiguous $150 \mathrm{MHz}$ block that the National Telecommunication and Information Administration (NTIA) identified as "fast track" band 3550$3700 \mathrm{MHz}$ for mobile broadband (MBB) and that the FCC calls Citizens Broadband Radio Service [15]. The 3550-3650 $\mathrm{MHz}$ spectrum is currently allocated for use by the US Department of Defense (DoD) ship-borne and ground based radar systems and the Fixed Satellite Service (FSS) receiveonly earth stations as shown in Fig.1. FSS operators have incumbency from $3600-3700 \mathrm{MHz}$ with additional protection requirement for adjacent band FSS sites operating in 3700$4200 \mathrm{MHz}$. In the 3650-3700 MHz spectrum band, there are protected DoD assets continued from a prior agreement, as well as the grandfathered commercial Wireless Broadband Service (WBS) users, which are only protected for five years at which point those systems become subject to CBRS rules as GAA or PA services. The FCC emphasize the role of the CBRS as an "innovation band" where spectrum can be assigned to commercial MBB systems like the third generation partnership program (3GPP) Long Term Evolution (LTE) on a shared basis with incumbent systems and promote a diversity of Heterogeneous Network (HetNet) technologies, particularly small cells. The sharing framework consists of three tiers: Incumbent Access (IA), Priority Access Licenses (PAL) and General Authorized Access (GAA) as shown in Fig.1. The technology neutrality of the CBRS will in particular play a role in the opportunistic GAA tier, opening up new access opportunities [16]. Rules are optimized for small cell use, but can also accommodate pointto-point and point-to-multipoint use, particularly in rural areas. 


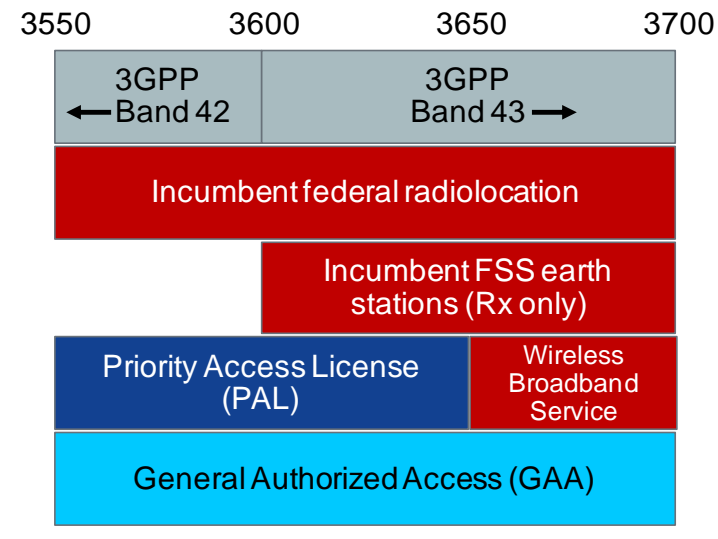

Figure 1. The US 3-tier authorization framework with the FCC's spectrum access models for $3550-3650 \mathrm{MHz}$ and $3650-3700 \mathrm{MHz}$ spectrum segments

The $P A$ users will obtain FCC licenses to operate up to a total of $70 \mathrm{MHz}$ of the $3550-3650 \mathrm{MHz}$ spectrum segment and are protected from harmful interference from the GAA operations. The PA users receive short term priority authorization to operate within designated geographic areas such as 3 year $10 \mathrm{MHz}$ unpaired channel in a single census track, awarded with competitive bidding. An applicant may apply for up to two consecutive three-year terms for any given PAL during the first application window. In order to ensure availability of PAL spectrum to at least two licensed users in the highest demand areas, licenses will be permitted to hold no more than four PALs in one census track at one, and no licenses are granted if there is only one applicant except in rural area. PALs are auctioned to the licensee within their service area but the specific frequencies are assigned by a SAS and they may be changed by a SAS, if necessary. At the end of its term, the PAL will be automatically terminated and may not be renewed.

The third GAA tier will operate under a licensed-by-rule framework throughout the $150 \mathrm{MHz}$ band with a minimum of $80 \mathrm{MHz}$ and maximum of $150 \mathrm{MHz}$ in each area, subject to incumbent and PAL tier activity. The GAA users have no interference protection from other CBRS users, while they must protect incumbents and PALs. GAA users may utilize unused PAL spectrum. This framework aims to facilitate the rapid deployment of compliant small cell devices while minimizing administrative costs and burdens on the public, licensees, and the FCC. The GAA is planned to provide a low-cost entry point into the CBRS band for a wide array of users and services, e.g., personal, small business and campus local hot spots, PAL offload during IA interruption, MBB capacity/offload, backhauling and WBS. The GAA users may use only certified, Commission approved CBRS devices (CBSD) and must register with a SAS with information required by the rules, e.g., operator ID, device ID and parameters, and geo-location information [17].

$C B S D$ s are fixed or portable base stations or access points, or networks of such stations and can only operate under the authority and management of a centralized the FCC selected SAS, which could be multiple, as shown in Fig. 2. In case CBSD is a managed network as in the typical case of MNOs, CBSD network includes the domain proxy (DP) and network management functionality. The DP could be a pure bidirectional information processing and routing engine or an intelligent mediation function, e.g., combining individual small cells of a mall or sports arena into a virtual base station entity that covers the complete mall or sports arena. The latter option allows flexible self-control and interference optimizations in such a network.

The $S A S$ assigns spectrum and determines and enforces maximum power levels dynamically to certified CBSDs at a specific geographic location and time, controls the interference environment and enforces protection criteria and exclusion zones to protect higher priority users. The SAS also takes care of registration, authentication and identification of user information and performs other functions as set forth in the FCC rules. As the IA users have primary spectrum rights at all times and in all areas over PA and GAA, all the CBSDs and End User Devices (EUDs) must be capable of two-way communications across the entire $3.5 \mathrm{GHz}$ band and discontinuing operation or changing frequencies at the direction of the SAS to protect the IA.

It is mandatory for all the CBSDs to protect the IA users in the band. Based on nature and critical requirements of the federal incumbent the FCC adopted rules to require Environmental Sensing Capabilities (ESCs) to be approved and detect incumbent radar activity in coastal areas and near inland military bases in and adjacent band before category B (47 dBm/10 MHz) CBSDs may be authorized in the 3550$3650 \mathrm{MHz}$ portion of the band. There will be strict rules and corresponding certification for the ESC component in order to ensure confidentiality of the sensitive military incumbent information [18]. When IA activity is detected, the ESC communicates that information to the SAS, which, if needed, could order commercial tier users to vacate a spectrum resource within 300 seconds in frequency, location, power, or time when proximity to federal IA radar presents a risk of harmful interference. Federal IA protection will be introduced in 2 phases: First, a large area of the country outside the static exclusion zones will be available after the SAS is the FCC approved and commercially available. At the second phase, exclusion zones will be converted to protection zones through the ESC system enabling the rest of the country, including major coastal areas, to become available. An ESC consists of one or more commercially operated networks of sensing devices that would be used to detect signals from federal radar systems in the vicinity of the exclusion zones. Additionally, a CBSD infrastructure based 
sensing could be considered under the strict operational security requirements. Prospective ESC operators must have their systems approved through the similar process as SASs and SAS administrators.

A SAS would obtain the FCC maintained information, about registered or licensed commercial users from the FCC databases, and exclusion zone information maintained by the NTIA. Functional architecture has option for the informing incumbent in-case the federal IA wants to inform the SAS ahead of plans to use the spectrum in some area, e.g., related to planned use of the spectrum.

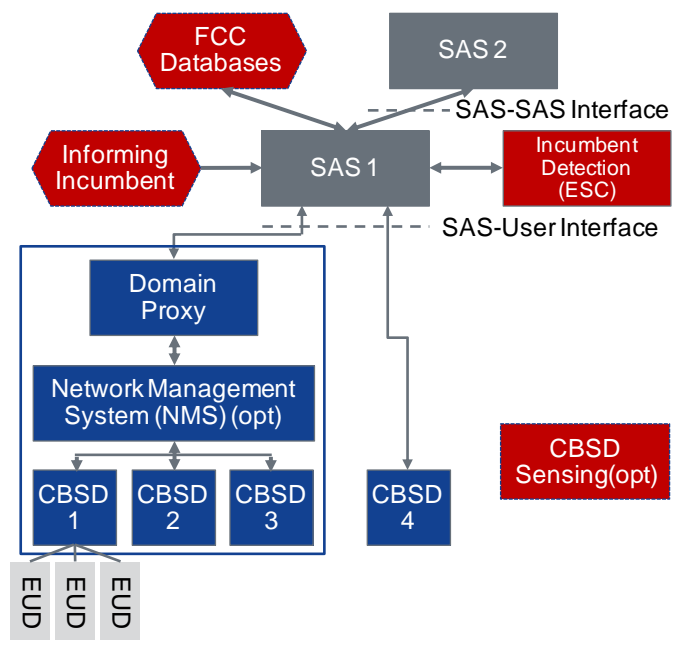

Figure 2. The CBRS functional architecture and key domains [19].

The GAA tier is planned to provide a low-cost opportunistic entry point into the CBRS band while the PAL system operations have to wait for the auction process. For the meanwhile, the FCC has encouraged multi-stakeholder groups to consider various issues raised by the rules. The Wireless Innovation Forum (Winn Forum) Spectrum Sharing Committee (SSC) [20] with representatives from the government, mobile broadband, wireless, Internet and defense ecosystems serve as a common standards body to support the development and advancement of spectrum sharing technologies with initial focus on the $3.5 \mathrm{GHz}$ targeted to allow early sharing trials of the CBRS within 2016. Committee does standardization work on functionality and architecture, requirements and interoperability, security, protocols and data models, and testing and verification [19] [25]. Base on these initial technical reports several CBRS field trials are ongoing, e.g., [26] and [27]. Furthermore, the 3GPP initiated work for the CBRS $3.5 \mathrm{GHz}$ band for LTE in the United States in June 2016, with the expected milestone for finalization of the band definition in December 2016 [28].

The US Government has initially identified an additional $2 \mathrm{GHz}$ of spectrum below $6 \mathrm{GHz}$ owned by federal users for future shared commercial use [29]. The success of the CBRS is critical to future federal-commercial spectrum sharing. Moreover, the FCC has already proposed the use of the three tier model and the SAS for $5 \mathrm{G}$ in several mmWave and cmWave bands [30]. This paves the way to make licensed spectrum sharing a third mainstream way of spectrum authorization to commercial users complementing traditional exclusive licensing and unlicensed spectrum access. The FCC has a vision to repeat $\mathrm{WiFi}$ success through lowering the entry barrier to QoS spectrum for new entrants and verticals, e.g., enterprise, utilities, healthcare, public safety, and smart cities. Based on our dynamic capability analysis, we discuss different antecedents, processes and outcomes that potentially motivate incumbents and other stakeholders to see spectrum sharing as an opportunity, and provide views for the regulatory and standardization bodies to be considered when further developing guidelines and policies for spectrum sharing, especially within the CBRS and the SAS.

\section{DYNAMIC CAPABILITIES IN CBRS}

\subsection{Theory of dynamic capabilities}

Strategic management literature is employing DCs to characterize the use of company resources in a rapidly changing environment in order to achieve value creation and capture. The DC approach facilitates the identification of company or industry specific processes that are critical to company evolution [31] in identifying new opportunities and organizing effectively and efficiently to embrace them. In practical use the DC concept can be divided into three domains: the antecedents (internal and external factors), the elements (contents, knowledge and processes), and the outcomes of DCs (linkage to economic performance and competitive advantage) [32].

Resources and capabilities can be conceptualized as hierarchical constructs. At the bottom of the hierarchy are resources, zero-order elements [31]. Operative capabilities, the first-order elements, skills required for utilizing resources, are higher in the hierarchy [33] followed by the second-order elements, core capabilities which are the critical for doing business [34]. In addition to having above discussed capabilities and being able to do something the third order dynamic capabilities [34] are needed to be able to create new ways of doing business and renewal. DCs influence the development and govern the rate of change of operational and core capabilities [35] in a systematic way containing patterned elements and involving learning [36]. 


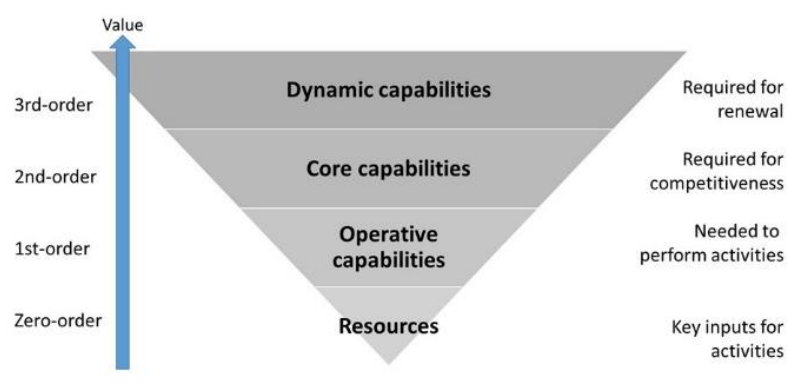

Figure 3. The dynamic capabilities framework.

The DC research scope has recently been widened from a specific company to inter-organizational, either networked or ecosystemic, relationships. For example, a DC can denote a company's capability to access and utilize partners' complementary resources and capabilities as an alternative for developing capabilities themselves or acquiring other organizations' capabilities [37]. In the collaborative DC context, Eriksson [38] pointed out the importance for stakeholders to continuously observe and assess partner activities and the value of the collaborative arrangement. Furthermore, it was found central that the concurrent activities and outputs of the partners are compatible and can be integrated with those of the focal firm. The DC framework has been applied to mobile communications and spectrum sharing in [39] to derive incentives for incumbent spectrum uses in the static/semi-static LSA concept. In this paper, we expand the work by applying DC to the more complex and dynamic CBRS concept.

\subsection{CBRS from the dynamic capabilities perspective}

Building on the definition of the CBRS, the SAS and the discussion on DCs, we identify five domains in the high level functional architecture where key stakeholders face the need for DCs, considering spectrum provisioning, utilization and its management:

- Incumbent Access system - the unused excess spectrum that the incumbent has access to,

- National Regulatory Authority - conditions, rules and incentives for sharing between the incumbent and the CBRS users,

- Spectrum Access System - database of the information regarding the sharing rules and availability of spectrum, Environmental Sensing Capabilities (ESCs) and a controller that enforces the sharing rules,

- CBSD access networks - the PA and the GAA networks to utilize the spectrum, including optional domain proxy and network management system (NMS), and
- End User devices - capable of two-way communications across the entire $3.5 \mathrm{GHz}$ band and operation on PA and/or GAA networks.

Table 1 presents these key functional architecture domains in detail, by looking at the antecedents, elements, and outcomes.

The incumbent access system and the excess spectrum are the points of departure for discussing the CBRS from the DC perspective. Due to incumbents' mandatory obligations and long life-cycle investments made in their systems, it is natural that they want to continue to use the spectrum they have access to. As an antecedent, incumbents' underutilization of spectrum combined with demand side spectrum crunch has led to a situation where the regulator is pressured to give a "price tag" to spectrum. However, there has to be enough desired spectrum available to be shared to attract investments. Also, control and appropriateness of value related rules over spectrum have to be clear and predictable for the incumbent. In the case of federal DoD incumbent, compatibility with military spectrum management objectives and procedures and long product lifecycles together with high relocation costs are important framing elements to consider. Spectrum sharing with the DoD is constrained by Operation Security (OPSEC), e.g., requirement for the ESC to be unable to geo-locate Naval radars more accurately than 65 miles. Such requirements can complicate the ESC design, inter-SAS cooperation, and SAS data retention policies preventing greater accuracy via cooperation and an adversary from learning patterns of movement. Regarding the key processes for the CBRS, the incumbent must be capable for identifying its own spectrum usage and reporting it as required by the FCC. In the case of federal incumbent, it is the responsibility of the ESC to detect usage non-cooperatively. Within the military domain, operational context, like technical user requirements are challenging to access.

The national regulatory authority with sharing rules can be seen essential for the success of CBRS. Political support for sharing, together with related legislation and regulation, can be identified as an antecedent for the CBRS. A sharing framework should find an appropriate balance between QoS guarantees and long-term availability, and short term licenses to enable successor uses to enter the band. On one hand, the increasing demand for mobile services and capacity and related need for additional spectrum, to be used under fair and transparent access rights, serves as a starting point. On the other hand, government direct budgetary requirements and incentives for entrepreneurs and economic growth should support this. It is important to make both the PAL and particularly the GAA available for early adopters as early as possible for service and business model trials. To speed up go-to-market from technology domain, the NRA has a role in facilitating inter-domain interference studies, e.g., exclusion 
zone analysis. Regarding the key processes, identifying additional bands for sharing, after initial $3.5 \mathrm{GHz}$ band, through long term planning and the establishment of a sharing arrangement and framework with incentives for both the PA and the GAA users, plays a crucial role. Enabling processes include improving federal spectrum management and utilization, and novel incentive processes, such as, incentive auctions, administrative pricing or spectrum currency. As an outcome, we identify the increased efficiency of spectrum use and more flexibility in spectrum use in particularly through the introduction of the third GAA tier. Promotion of competition with lower entry barrier to access spectrum allows an NRA to empower entrepreneurs to innovate in vertical markets and intensify small local operators in urban hot spots and rural areas. Stable framework to shorter transition times, better valuation of shared spectrum and revenue from annual payments can be seen as the outcomes of the CBRS.

The $S A S$, and SAS and ESC administrators form the third area for DCs in the CBRS. As a mandatory antecedent, we can consider that a standardized functional architecture, with defined interfaces, data models and protocols must exist and the required level of operational and communication security must be maintained. In the overall system, the SAS plays central role in inter-operability and in controlling the degree of dynamics and complexity. The SAS with deep, near realtime insight into all CBSDs and CBSD networks, requires core competencies in scalable big data and analytics capabilities and deep knowledge and experience in radio propagation modeling. SAS operators and ESC operators need not be the same entities, and need to build trust and collaboration in inter-machine and inter-firm levels. Particularly, the MNOs data privacy concerns related to the exposure of their sensitive network configuration information can have negative impact on the inter-SAS coordination, protection mechanisms for IAs, and SAS-CBSD interface design. The key processes in this area founded on employing the NRA rules in coordination with other SASs are related to making the spectrum available for sharing. In coordination with the incumbent operations through database and the ESC sensing, a SAS should predict and manage aggregate interference protections for IA users and for PA service area from other PA devices and from GAA devices, detect opportunities, and dynamically assign frequency channels and enforce changes to CBSD access networks accordingly. Particular challenge for the SAS function will be the interference originating from distinct networks deployed in neighboring census tracks by different users without coordination. Consequently, the SAS service could be expanded in the future to cover the PAL and the GAA coexistence management, providing a list of preferred channels based on interference estimations and measurements. The SAS system should address operations (OPSEC), data and communication (COMSEC) security towards all involved stakeholders for authentication, authorization and encryption of SAS-SAS, SAS-Proxy and SAS-CBSD interface. Making spectrum accessible is not enough; the underutilized assets need to move within the ecosystem. The trust is the trigger of collaborative shared consumption that makes system grow and scale. The creation of a critical mass ecosystem with positive network effects is important for the CBRS with new context model based spectrum administrator and broker roles, where collected user and context data could be utilized through data monetization business processes. The centralized SAS has been considered as the basic vehicle to accomplish trust in recent sharing models with a principal role to simplify and automate the inter-firm relationships and processes in the CBRS. The SAS enables any number of incumbents and secondary users to share spectrum so that each operator-incumbent pair does not have to define and enforce unique sharing agreements.

At the CBSD access network level, exclusive access to QoS spectrum assets over long period has been essential antecedent for a MNO's strategic position in the market. Uncertainty and risks related to regulation in timing, term, licenses and flexibility create exposure and risk for an MNO to proceed with the investment. Furthermore, there is an impact on exclusive spectrum licensing model, availability and valuation in the future. Spectrum valuation is a complex issue influenced by factors from strategy, technologies, market position and competition, economics and regulatory decisions. As antecedents for the CBSD network we identify harmonization of technology, utilization of existing technology and spectrum assets, the cellular infrastructure topology, scalable ecosystem and existing market positions of operators. For existing MNOs leveraging the 3GPP LTE evolution as well as for the whole emerging CBRS ecosystem harmonization of radios (e.g., power and out-of-bandemission limits) and spectrum (alignment with the 3GPP bands) over the PAL and the GAA is important to achieve scale effects. To enable new low cost base station and access point products to operate, particularly on the GAA tier, it is essential to combine them with access to backhaul bandwidth, possibly needed for virtualized core network functionality. At the managed network domain proxy level we identify as an additional antecedent the near real-time network management system for CBSD element management and cross layer and co-existence interference resolution in addition to load balancing between demand and current network load. Current suspension and relocation duration requirement of 300 seconds will potentially mean introduction of a new technology.

The key processes we see are the configuration and optimization of the CBSDs and CBSD network, according to availability, quality, potential interference level and rules and conditions of the sharing framework guided by the SAS in order to avoid any harmful interference. This requires a new CBSD-SAS interface and implementation of protocol work 
flows. In the operation phase, initial network planning based on CBRS spectrum availability and existing network assets will be automatically configured and optimized according to intra CBRS band (PAL and GAA) and inter-band (HetNet layers) availability and demand, e.g., through utilizing novel customer experience management (CEM) and Self Organizing Network (SON) features embedded in operations support subsystem (OSS). For a CBSD operator, the entering to local small cell and/or vertical business domains calls for dynamic capabilities in business strategy and modeling across domains and verticals.

At this level, the disruptive outcome is the unbundling investment in spectrum, network infrastructure and services flexibility in the local spectrum use. More system bandwidth in dense area deployment means fewer base stations to meet the growing demand. Increased dynamics and flexibility enable faster access to low cost extra capacity in dense urban hot spots as well as for rural coverage when and where needed. For established MNOs the CBRS enables an additional spectrum layer for optimizing the use across all their spectrum assets. Additional PAL provides exclusive use spectrum, and GAA can be used for complementing WiFi offloading with novel LTE technologies such as 3GPP as License Assisted Access (LAA) [40]. Using LAA, operators could benefit from the additional capacity available from the GAA, particularly in hotspots and corporate environments. GAA layer would also provide cable operators, local small cell networks, MVNOs, WISPs, and Internet players an opportunity to access spectrum. The technology neutrality of the CBRS will play a role particularly in the GAA tier. IEEE based technologies will be complemented with a novel standalone version of LTE unlicensed (LTE-U) such as MulteFire proposal [41], which does not require a primary cell anchor in licensed spectrum. It aims on one hand to broaden the LTE ecosystem to entities that may not own licensed spectrum, and on the other hand, for MNOs to benefit from it through offloading and augmenting their mobile networks. Furthermore, the three tier model offers network operators unprecedented flexibility and scalability through the ability for to move between the PA and GAA tiers. This allows for the use of much shorter leasing periods, 3 years, without requiring a lessee to forgo their investment if their lease does not renew via simply converting from PA to GAA tier. For a new market entrant this enables to try out their new service utilizing the GAA tier without having to invest in spectrum with future option to choose buy a PA license when / where needed depending on the market and interference protection needs. In the system level this flexibility and scalability between tiers combined with the secondary market provisions will improve spectrum efficiency in capacity, and particularly in value as spectrum can be regularly re-allocated to the most valuable use. New technology introduction should be continuously assessed in relation with added complexity and transaction costs.

Finally, at the end user device level common antecedent is harmonization and scale of technology and spectrum. On one hand, leveraging existing technology in the CBRS ecosystem will reduce strategic risk and speed up market opening and scaling. As an example, the EUD emission limits that are compliant with the 3GPP specifications to enable the use of existing $3.5 \mathrm{GHz}$ 3GPP Bands $42 \& 43$ in the United States. On the other hand, the introduction of new full CBRS band will imply a need for standardization supported by related R\&D. Discussed intra-CBRS band flexibility can incentivize the development and deployment of interference tolerant receivers in CBRS radios to save the cost of a PA license, which also leads to improved spectrum efficiency. Standardization process of possible new requirements and bands is central in achieving positive outcomes like timely availability of terminals and the LTE ecosystem scale up. Furthermore, standardization would help in avoiding potential negative impacts related to cost, complexity and the GAA device co-existence interference. 
Table 1. CBRS in the dynamic capability view

\begin{tabular}{|c|c|c|c|}
\hline & Antecedents & Elements & Outcomes \\
\hline $\begin{array}{l}\text { Incumbent } \\
\text { Access }\end{array}$ & $\begin{array}{l}\text { - underutilized spectrum assets } \\
\text { - governmental pressure on defense } \\
\text { expenditure } \\
\text { - mandatory to continue critical } \\
\text { operations } \\
\text { - OPSEC constraints } \\
\text { - long product life-cycles and high } \\
\text { relocation costs }\end{array}$ & $\begin{array}{l}\text { - identifying and offering desirable } \\
\text { spectrum assets for sharing } \\
\text { - iteratively phased predictable approach } \\
\text { to novel spectrum management } \\
\text { concepts } \\
\text { - administrative processes in } \\
\text { procurement and operations domains }\end{array}$ & $\begin{array}{l}\text { - retain rights and OPSEC control to } \\
\text { the spectrum (no re-allocation) with } \\
\text { interference protection } \\
\text { - save on spectrum fees / create } \\
\text { revenues } \\
\text { - demonstrate ability and willingness } \\
\text { to contribute } \\
\text { - relocation and research fund for } \\
\text { technology renewal }\end{array}$ \\
\hline $\begin{array}{l}\text { National } \\
\text { Regulatory } \\
\text { Authority }\end{array}$ & $\begin{array}{l}\text { - increasing demand for mobile } \\
\text { services and capacity } \\
\text { - supporting legislation, regulation and } \\
\text { political willingness } \\
\text { - government direct budgetary } \\
\text { requirements and incentives for } \\
\text { entrepreneurs and economic growth } \\
\text { - inter-domain interference studies i.e. } \\
\text { exclusion zone analysis }\end{array}$ & $\begin{array}{l}\text { - identifying specific bands for sharing } \\
\text { through long term planning } \\
\text { - improving federal spectrum } \\
\text { management and utilization } \\
\text { - incentive process utilizing, e.g., } \\
\text { incentive auctions, administrative } \\
\text { pricing or spectrum currency } \\
\text { - sharing arrangement and framework } \\
\text { rules and conditions with incentives } \\
\text { for both PAL and GAA users }\end{array}$ & $\begin{array}{l}\text { - further efficiency with GAA } \\
\text { - promote competition with lower } \\
\text { entry barrier to access spectrum } \\
\text { - empower entrepreneurs in verticals } \\
\text { - provide incentives for local operators } \\
\text { - stable framework } \\
\text { - better valuation of shared spectrum } \\
\text { - shorter transition times } \\
\text { - revenue from annual payments }\end{array}$ \\
\hline $\begin{array}{l}\text { Spectrum } \\
\text { Access System }\end{array}$ & $\begin{array}{l}\text { - standardized functional architecture, } \\
\text { interfaces, data models and protocols } \\
\text { - operational, data and communication } \\
\text { security; data privacy } \\
\text { - inter-operability and verification } \\
\text { - controlled degree of dynamics and } \\
\text { complexity } \\
\text { - deep near real time insight into CBRS } \\
\text { networks } \\
\text { - scalable big data and analytics } \\
\text { capabilities } \\
\text { - experience of radio propagation } \\
\text { modeling }\end{array}$ & $\begin{array}{l}\text { - employing NRA rules in coordination } \\
\text { with other SASs } \\
\text { - coordination with incumbent } \\
\text { operations } \\
\text { - incumbent ESC sensing (could be } \\
\text { independent sensor network) } \\
\text { - predict and manage interference } \\
\text { - opportunity detection and dynamic } \\
\text { frequency assignment (interference, } \\
\text { co-existence) } \\
\text { - CBSD authorization and usage } \\
\text { monitoring }\end{array}$ & $\begin{array}{l}\text { - automatization of inter-firm } \\
\text { relationships in the CBRS } \\
\text { - incumbent protection } \\
\text { - Quality of Service certainty and } \\
\text { guaranteed spectrum for PA users } \\
\text { - optimize spectrum availability for } \\
\text { GAA users } \\
\text { - monitor and trace the use of } \\
\text { spectrum, possible harmful } \\
\text { interference, and other phenomena } \\
\text { - possibility to use data for license fees } \\
\text { and value added services } \\
\text { - real option to move towards } \\
\text { spectrum aggregator/ broker role }\end{array}$ \\
\hline $\begin{array}{l}\text { End } \\
\text { User } \\
\text { Device }\end{array}$ & $\begin{array}{l}\text { - scale of technology } \\
\text { - device ecosystem } \\
\text { - harmonization of spectrum } \\
\text { - full CBRS spectrum coverage }\end{array}$ & $\begin{array}{l}\text { - standardization of possible new } \\
\text { requirements and bands }\end{array}$ & $\begin{array}{l}\text { - timely availability of terminals and } \\
\text { potential impact on cost and } \\
\text { complexity } \\
\text { - LTE ecosystem scale up } \\
\text { - GAA devices create co-existence } \\
\text { interference concerns }\end{array}$ \\
\hline
\end{tabular}




\subsection{Summary of value creation and value capture opportunities}

This section summarizes the outcomes of Dynamic Capabilities by identifying the sources of value creation and capture by key stakeholders stemming from the use of internal and external resources overcoming the internal and external resistance for co-creation and co-capturing value in exchange.

The CBRS three tiered regulatory approach can disruptively unbundle investment in spectrum, network infrastructure, services and location, utilizing introduced flexibility and scalability in regulation and spectrum management implemented in the SAS. The regulatory lighttouch leasing process will make the spectrum use rights held by PA licensees available in secondary markets, driven by specific local business needs. Furthermore, access to free or low cost spectrum with lower initial annuity payments for spectrum rights enables local 'pro-competitive' deployments. Access and deployment of the underutilized assets ondemand is essential for entrepreneurs to test the novel services and generate revenue early. The CBRS spectrum sharing business models are hosted through platforms and automatized processes that enable a more precise, real-time measurement of available capacity, and the ability to dynamically making that capacity accessible. Dynamic adaptability to short-term changes and automatic configuration of radio infrastructure is the key differentiator to semi-static sharing concepts, e.g., the LSA.

Value of the shared spectrum resources is highly dependent on the availability, liquidity and predictability of this idling capacity. The transaction costs such as searching for an opportunity, contracting process, satisfying government requirements, building trust and operational costs may deprive economies of scale and scope, raise costs and hamper innovation, and could create a strong barrier in terms of value scalability. Current concerns include considerations for incumbent OPSEC and operators' data privacy necessarily, which can decrease spectrum sharing efficiency and complicate inter-organization cooperation. After having a willing incumbent and licensee, the final requirement for the successful spectrum sharing service is acceptably low 'pay as you grow' transaction costs. These costs can be reduced by spectrum brokers and flexibly light touch regulatory approach in order to streamline processes for transferring rights. Combined with ability to test a new service early and accelerate time to market will enable scale.

While the third opportunistic GAA layer offers Wi-Fi ecosystem type innovation environment, the availability, and particularly the QoS is not guaranteed. This has limited operators' interest, based on traditional business models with need for the high upfront investments. On dense urban environment, new business model designs and revenue structures could emerge combining spectrum with other shared assets, e.g., small cell hosted solution-as-a-service (SCaaS), advertisement \& transaction based models and enabling new vertical segments within IoT. The complexity of CBRS introduces new independent or integrated roles to the ecosystem related to the SAS administration, ESC sensing operator and future spectrum broker.

Simplicity of the offer built around user knowledge driven 'demand pull' with faster efficient access to cuttingedge systems and services from inception is critical in differentiating and achieving market advantage with innovative business model designs. In particular, new entrants from Internet domain utilizing content, context and commerce business models would like to see the CBRS broadband as a utility, transparent and non-exclusive basis. Instead of connectivity, their business models will be based on capturing value through of digital ads, cloud, retail or content, while creating value with free access. In addition to provide mandatory spectrum availability information exchange, the SAS administrators can capture value through brokering advanced information regarding the quality, liquidity and predictability of the shared spectrum resources based on information from both the incumbents and sharing users. These value added services will be framed by regulatory action, and their value will increase with the number of users, creating a positive network externality. The SAS platform in itself lowers the entry barriers, and have inbuilt incentives to collaborate efficiently.

Shared spectrum local small cell deployments in all of the sharing concepts scale out ecosystems, as small cells will attach to structures and building assets not owned by traditional operator. This creates additional opportunities for sharing and collaboration between operators and various companies like infrastructure owners and providers, venue owners, enterprise and utility service companies.

\section{TECHNOLOGY ENABLERS}

In this chapter, the DC analysis is complemented with assessment of the technology enabler for key processes: spectrum provisioning, utilization and its management in the five functional architecture domains: IA, NRA, SAS, CBSD and EUD. Table 2 summarizes these antecedents, by looking at the key technologies, their availability and further development needs in order to exploit CBRS spectrum sharing in mobile broadband networks.

\subsection{Incumbent access system}

Primary antecedent for the overall CBRS sharing is the IA's knowledge of its protection requirements, including the acceptable interference level, from prior sharing studies. The other antecedent is to register own operational parameters with the SAS for non-ESC requiring IA. In the case of DoD, 
where ESC is considered, there are strict limitations on how the information on the IA should be handled. For ESC, the requirements on both sensitivity and periodicity of sensing need to be defined, as they have an effect on both the deployment and use of the ESC system. As the CBRS framework enables sharing rules and conditions to be flexibly changed through the SAS, it is important for an IA to be able to estimate the future enhancements of its own technology, for example, cognitive radar systems and interference tolerant receivers.

\subsection{National regulatory authority}

Existing databases on spectrum assignments and usage form the basis for the NRA operations. Database can be maintained by the NRA itself or a trusted third party. SASs should be capable of receiving and responding to interference complaints from the FSS earth station licensees. Upon such complaint it is essential for the predictability and trust that the NRA has defined enforcement methods and tools to handle possible interference incidents. E.g., in the case of the CBRS, the FCC is working with the industry in order to define a common propagation model for FSS earth station protection to be used in SASs, and inter-domain interference studies, e.g., exclusion zone analysis for the first phase service. The NRA provides methods and tools for inter-operability, verification and certification. Auction mechanisms and tools for the initial PA licensing should be further developed to facilitate the PAL leasing in the SAS [14]. In addition to more traditional PAL licensing, rules for real-time authorization of both PA and GAA users done by the SAS, need to be defined by the NRA. This may include priorities amongst users or spectrum bands.

The regulatory and standardization actions needed with regulated or highly political incumbents' ecosystem will potentially limit the scalability. As one solution to tackle such issues, the novel Blockchain (BC) [42] technology, the 'decentralized and shared ledger' that underpins bitcoin, has emerged as an object of intense research in the financial services industry and beyond. Multipath connections inherent to $\mathrm{P} 2 \mathrm{P}$ network can create a potential barrier for the regulation. The $\mathrm{BC}$ has potential to improve accounting for value in the measurement, processing and communicating of transaction and financial information about stakeholders. In the CBRS regulatory enforcement processes, a BC regulatory agent can participate as a node, and may have rights to inspect and record the data, participate the voting, and, e.g., stop transactions. Furthermore, two-way references to 'realworld' contract could be utilized as dual integrations to strengthen legal enforcement. As an open source concept, the $\mathrm{BC}$ can flexibly innovate, iterate and improve as well as adapt in the regulatory environment.

\subsection{Spectrum Access System}

A essential technology enabler for the CBRS system architecture is the detailed standardized protocols for data and communications across the various open interfaces within the system between SASs, and between SASs and incumbents, ESC, and CBSD users. The communications security policies govern a SAS and CBSD communications interfaces, and describes a Public Key Infrastructure (PKI) which governs communications within the CBRS ecosystem and provide authentication and authorization for messages exchanged within the SAS ecosystem.

Key technology enablers are the scalable, high availability platform for CBRS databases, big data, analytics and future machine learning. These enablers have synergies with context Internet business domain technology platforms. The principal function of the SAS is channel management and dynamic channel allocation algorithms for assigning spectrum to CBRS users. Implementation of common propagation models should be complemented with scalable radio mapping tool and algorithms for interference calculations. A SAS operator could provide value added services based on advanced 3D radio propagation map, spectrum analytics tools and information on the operators' NMS and SON network data. In order to mitigate the negative impacts from multiple signals, possibly across multiple air interface technologies, occupying the same and adjacent channels, a SAS could leverage spectrum analytics and SON features with the DP and NMS, depending on SAS operator's agreement with the CBSD operator. Key objectives for coexistence management functionality in the early GAA deployments could consist of coexistence of different TDLTE operators in same geographical area, coexistence of different LTE variants, e.g., TD-LTE and LAA and MulteFire in same geographical area while providing fair access to spectrum and ensuring efficient utilization of available spectrum.

The FCC rules [14] call for the establishment of an ESC monitoring network in concert with a SAS to protect offshore radar receivers. ESC also can apply to ground-based radars. Higher power outdoor category B CBSDs will only be authorized to use spectrum after an ESC is approved and commercially deployed. SAS and ESC operators may be the same or two separate entities. As a future option, CBSDs could participate ESC through CBSD radio integrated sensing function [43].

The FCC $2^{\text {nd }} \mathrm{R} \& \mathrm{O}$ [14] finalized rules for secondary spectrum markets. The Light Touch Leasing for PALs option streamlines (subject to oversight) the process which could greatly enhance the robustness of the secondary spectrum market, and enables introduction of a SAS spectrum brokering functionality. The above discussed Blockchain [44] technology could be used to enable decentralized low transaction cost micro transactions in the SAS. It has potential to offer a novel way for a SAS to perform 'dynamic 
policy management' in a way that is secure, transparent, highly resistant to outages, auditable, and efficient [45]. Furthermore, the BC may drop many transaction costs related to search, contracting, enforcement, and making payments. As such, it offers the possibility of disrupting financial services, accounting and auditing, and enabling new business models through enabling sharing economy based future spectrum sharing regulation innovations [44].

\subsection{CBSD radio access network}

In case CBSDs are part of a managed network, as in the typical case of MNOs, operator network will include the domain proxy and network management systems functionality. Functional split between CBSD radios, DP, and NMS is highly dependent on the operator type and deployment scenario.

In the preparatory network provision phase, nominal CBRS network radio planning and dimensioning for the business case are carried out. Dynamic CBRS calls for more dynamic spectrum opportunity detection, evaluation, and decision making tool as a counterpart for the future SAS spectrum broker engine. In the managed CBSD network use case, operators are optimally positioned to utilize their existing NMS and SON solutions. HetNet SON functionalities could be leveraged, e.g., in automatizing the initialization and optimization of cell parameters, dynamically adapting the network in response to radio environment and network topology changes, optimizing cells bordering, optimizing operations during special events and performing actions for energy saving. Furthermore, in order to perform the closed-loop SON operations, SON platform maintains a database of all of the cells within the network as well the relationships among the cells based on measurements within the network and dynamically computes clusters relationships among cells. These cell level relationships are essential for the automated optimization of neighbor lists, Layer Management Strategy (LMS) enforcement, network border area management, handover parameters, reuse codes, control of Remote Electrical Tilting (RET) antenna settings, Physical Random Access Channel (PRACH) parameters, enhanced Inter-Cell Interference Coordination (eICIC) [43] and [46], etc. Several methods for the actual ICIC have been proposed as vendor specific solutions. The ICIC is handling the interference between UE resource allocation in each base station enabled by information exchange between base stations, e.g., by High Interference Indicator (HII) and Overload Indicator (OI). LMS Enforcement Module defines and enforces operatorspecific rules for managing transition between layers (technologies, bands, frequencies, vendors, indoor/outdoor, voice/data service, cell types, etc.). Network border area management manages regional external cells and their link to real cells and adjacencies across vendors and technologies.
Mobility Load Balancing (MLB) continuously monitors and identifies congested cells, and modifies parameters to offload traffic to surrounding cells via HO thresholds, reselection thresholds and/or power configuration. In traffic steering, awareness of distribution of class of subscribers within cells within the network over time could be used within SON Modules to optimize the network in line with operator priorities for their defined classes of service. In the smart antenna Beam steering SON module, instead of locking down or reducing power of the cell, radiation pattern per cell can be modified in order to prevent interference to the incumbent, and moreover to automatically adjust the sizes and positions of the cells to better utilize the shared spectrum to serve nonuniform demand from users across cell area. Moreover, novel smart antenna radio architectures enable integrated sensing solution that provides extra protection towards interference, improves accuracy, and reduces the area of the restriction zones [47].

Deploying the Domain Proxy element within the SON framework enables to leverage the deep RAN knowledge, history and status already existing within the NMS and SON. Additionally, when spectrum changes, e.g., allocation or deallocation, do occur the SON will dynamically optimize the network, e.g., adjust the inter and intra site/frequency/RAT neighbor relations to ensure continued optimal network performance. Furthermore, having a SAS and DP elements in the same domain enables utilization of cell relationships and maps based on real network measurements. This will provide superior results compared to using an embedded radio environment prediction engine, as it is based on what is actually occurring within the network instead of predictions based on channel propagation models. This approach is also far less computation-intensive than performing embedded RF predictions. For constrained CBRS use case with limited SON functionalities or in case CBSD operator is not willing to provide detailed network information to the SAS, the SAS will revert to the embedded RF prediction approach for those CBSDs.

Dynamism requirements for the CBSD radio access system is set by the FCC vacation rules [14] ordering a CBSD to vacate spectrum within 300 seconds of an incumbent detection or notification by a federal incumbent user, and furthermore, to comply with any SAS commands to cease transmission, change frequencies or power levels within 60 seconds. These requirements are critical for the CBSD netwrok implementation scenarios and under studies and validation in the first CBRS field trials. The key DP implementation question is whether strict evacuation time requirements could be met with existing NMS SON based solution or will this require NMS bypass, e.g., implementing the SAS-CBSD interfacing protocols and element management functionalities into the CBSD base stations [26].

For the key enabling CBSD radio technologies, global harmonization is essential to achieve economies of scale in 
both the CBRS band and across all other aggregated/balanced/steered spectrum bands. There will be two types of CBSDs: Category A CBSDs have lower power, and are limited to indoor or low-height outdoor operations, and Category B CBSDs have higher power and are limited to outdoor operation. Category B CBSDs will be authorized to use spectrum after an ESC is approved and commercially deployed.

For the opportunistic GAA layer and stand-alone deployments recent LTE unlicensed 3GPP standards are offering new technology options. LTE-U and LAA utilize Carrier Aggregation [48] in the downlink to combine LTE in unlicensed/shared spectrum with LTE in the licensed band [40]. For alternative operators or local hosted deployments without existing infrastructure on a licensed carrier, MulteFire based on LAA downlink and Release 14 enhanced-LAA (eLAA) for uplink will offer an alternative without a licensed spectrum anchor in the future [41].

In order to be future proof, CBSD should be upgradeable to add support, e.g., for advanced coexistence features. Further LTE functionalities to be developed include a method for achieving time sync between CBSDs in same cluster and across different clusters, and a mechanism to align TDD configuration parameter across different deployments to minimize guard band requirement.

In a network level, Network Function Virtualization (NFV) techniques lower the entry barrier to new operators, as network functions (elements) like the Evolved Packet Core (EPC) and NMS could be deployed and acquired as a service. Furthermore, dedicated virtual sub-networks, so called network slices can have different combinations to provide exactly those functionalities that are needed for different industries and their diverse use cases.

\subsection{End User Devices}

As for any new radio technology, the device availability for the band through harmonization of the band globally is essential. Reusing 3GPP bands 42 and 43 could be considered as an intermediate solution, though it will create problems with legacy band 42 capable devices not meeting the $3.5 \mathrm{GHz}$ FCC emission requirements. Moreover, dedicated CBRS band EUDs will provide better uniformity in device certification and testing [28].

All the CBSDs and EUDs must be capable of two-way communications across the entire $3.5 \mathrm{GHz}$ band. Furthermore, it would be beneficial to have multi-band multimode support to enable continuous QoS provision, requiring wideband transceiver architectures and chipsets. Furthermore, Carrier Aggregation band combinations (BCC) with licensed [48] or unlicensed band, e.g., through LTEWiFi RAN level aggregation (LWA) [49] will improve the QoE.
Table 2. Technology enablers for the CBRS

\begin{tabular}{|c|c|}
\hline Domain & Technology enablers \\
\hline $\begin{array}{l}\text { Incumbent } \\
\text { Access }\end{array}$ & $\begin{array}{l}\text { protection requirements from prior sharing } \\
\text { studies } \\
\text { - registration of own operational parameters } \\
\text { envisage the future enhancements of own } \\
\text { technology, e.g., Cognitive radar systems and } \\
\text { interference-tolerant receivers. }\end{array}$ \\
\hline $\begin{array}{l}\text { National } \\
\text { Regulatory } \\
\text { Authority }\end{array}$ & $\begin{array}{l}\text { - existing databases on spectrum assignments and } \\
\text { usage } \\
\text { interference incidents } \\
\text { - research for common propagation model for } \\
\text { SASs } \\
\text { - facilitates inter-domain interference studies, i.e., } \\
\text { exclusion zone analysis for initial service } \\
\text { - methods and tools for inter-operability, } \\
\text { verification and certification } \\
\text { - auction mechanisms and tools for the initial PA } \\
\text { licensing, and further development for the PAL } \\
\text { leasing in SAS } \\
\text { - rules for real-time authorization } \\
\text { Blockchain enabled solution to lower transaction } \\
\text { cost hurdle, build trust and enable sharing } \\
\text { economy regulatory innovation }\end{array}$ \\
\hline $\begin{array}{l}\text { Spectrum } \\
\text { Access } \\
\text { System }\end{array}$ & $\begin{array}{l}\text { - standardized functional architecture, interfaces, } \\
\text { data models and protocols } \\
\text { - operational and communication security } \\
\text { - scalable and high availability big data and } \\
\text { analytics capabilities related to spectrum and } \\
\text { databases } \\
\text { - scalable radio mapping, spectrum analytics and } \\
\text { algorithms for interference calculations } \\
\text { - advanced 3D radio propagation models for value } \\
\text { added services, e.g., for GAA co-existence } \\
\text { - dynamic channel allocation algorithms and } \\
\text { management functions } \\
\text { - ESC spectrum sensing network with SAS or in } \\
\text { collaboration with a ESC operator } \\
\text { - integration/utilization of spectrum analytics and } \\
\text { SON features } \\
\text { - spectrum broker functionality for the PAL } \\
\text { leasing } \\
\text { Blockchain enabling low transaction cost } \\
\text { microtransactions }\end{array}$ \\
\hline $\begin{array}{l}\text { CBSD } \\
\text { access } \\
\text { networks }\end{array}$ & $\begin{array}{l}\text { - spectrum opportunity detection, valuation and } \\
\text { decision making engine counterpart to SAS } \\
\text { broker } \\
\text { - near real time network element management } \\
\text { system for managed CBSD, e.g., lock/unlock, } \\
\text { frequency, power and antenna control. } \\
\text { - SON, e.g., spectrum analytics, layer } \\
\text { management strategy, load balancing, traffic } \\
\text { steering } \\
\text { - smart antenna beam steering } \\
\text { - Domain Proxy functionality } \\
\text { - network measurements capability, optionally } \\
\text { with collaborative sensing for the ESC } \\
\text { harmonization of radio technology, e.g., } \\
\text { spectrum bands, power and OOBE limits }\end{array}$ \\
\hline
\end{tabular}




\begin{tabular}{|ll|}
\hline & - interference tolerant receivers \\
& - managed network interference resolution \\
& capabilities \\
& - LTE unlicensed technologies, LAA \\
& - nulteFire standalone and neutral hosting radios \\
& - network slicing \\
\hline End & - utilization of harmonized spectrum bands to \\
User & scale the device ecosystem \\
Device & wide band radios to two-way communications \\
& across the entire 3.5 GHz CBRS band \\
& - multiband support for continuous QoS \\
& - Carrier Aggregation band combinations with \\
& LTE or WiFi LWA band for QoE \\
\hline
\end{tabular}

\section{CONLUSIONS}

This paper has presented a framework for analyzing technology and business enablers and sources of competitive advantage for the CBRS functional architecture among key stakeholders, in order to capture the potential benefits and the framing elements of the CBRS concept. By using the dynamic capability approach, we have identified the antecedents, elements, and outcomes of the CBRS in the five basic functional domains for spectrum sharing between users. Building on this analysis, we have provided some guidance for stakeholders about technology enablers, sources of value creation and actions to further promote spectrum sharing in the form of the CRBS. The DC analysis indicated the key role of the regulator in creating a sharing framework with incentives for all the key stakeholders with different operational and business requirements and enabling scaling ecosystem. Particularly, realizing and fine-tuning incumbent spectrum users' incentives and facilitating the restriction of the OPSEC and data privacy constraints could be very helpful in implementing CBRS on an additional spectrum for future shared commercial use. By pointing out the DCs involved in the CBRS, this paper showed how CBRS could be used to complement and improve current mobile broadband services and enable the entrance of new services and stakeholders particularly in local area and across verticals. This would be, on one hand, valuable to citizens and novel customers in verticals, and, on the other hand, beneficial for incumbents, e.g., by avoiding re-allocations, by providing additional revenues, or by lowering spectrum fees. Furthermore, incentives triggered by the CBRS may contribute to transition from administrative to market-based dynamic spectrum management. Increased system dynamics in spectrum sharing will introduce a need for big data analytics, efficient microtransaction, and near real time network management capabilities. Technology harmonization in spectrum and radios with dominant ecosystems will be essential to ensure economies of scale and fast time to market.
The successful deployment of the CBRS framework will significantly improve the efficiency of the spectrum use, influence the management approach of other spectrum bands and create new business opportunities. This calls for a collaborative effort from the government, industry and academia to build dynamic capabilities and technology enablers needed to incubate and accelerate the development the CBRS and the SAS.

\section{ACKNOWLEDGMENT}

This work has been done in the Local Area Spectrum Sharing (LASS) and the Cognitive Radio Trial Environment (CORE++) research projects within the 5G programs of Tekes - the Finnish Funding Agency for Technology and Innovation. The authors would like to acknowledge the project consortium members: VTT Technical Research Centre of Finland, University of Oulu, Centria University of Applied Sciences, Turku University of Applied Sciences, Anite, Bittium, PehuTec, Finnish Defense Forces, Finnish Communications Regulatory Authority and Tekes.

\section{REFERENCES}

[1] Cisco white paper, "Visual Networking Index: Global Mobile Data Traffic Forecast Update, 2015-2020. [Online]. Available: http://www.cisco.com/c/en/us/solutions/collateral/serviceprovider/visual-networking-index-vni/mobile-white-paper-c11520862.html. Feb. 2016.

[2] ITU Report M.2290-0, "Future spectrum requirements estimate for terrestrial IMT," 2014.

[3] The White House, President's Council of Advisors on Science and Technology (PCAST) Report, "Realizing the Full Potential of Government-Held Spectrum to Spur Economic Growth,” July 2012.

[4] The FCC, "The $3.5 \mathrm{GHz}$ report and order and second further notice of proposed rulemaking," [Online]. Available: http://transition.fcc.gov/Daily_Releases/Daily_Business/2015/db0421 /FCC-15-47A1.pdf, April 2015.

[5] The FCC, "White Spaces," [Online]. Available: http://www.fcc.gov/topic/white-space

[6] ECC Report 205, "Licensed Shared Access," 2014.

[7] J. Chapin and W. Lehr, "Cognitive radios for dynamic spectrum access - The path to market success for dynamic spectrum access technology," IEEE Commun. Mag., vol. 45, no. 5, pp. 96-103, 2007.

[8] M. Matinmikko et al., "Business benefits of Licensed Shared Access (LSA) for key stakeholders," In O. Holland, H. Bogucka \& A. Medeisis (eds.) Opportunistic Spectrum Sharing and White Space Access: The Practical Reality. John Wiley \& Sons, 2015.

[9] P. Ahokangas, M. Matinmikko, S. Yrjölä, H. Okkonen and T. Casey, “ "Simple rules" for mobile network operators' strategic choices in future cognitive spectrum sharing networks," IEEE Wireless Communications, vol.20, no.2, pp. 20-26, 2013.

[10] P. Ballon and S. Delaere, "Flexible spectrum and future business models for the mobile industry. Telematics and Informatics 26(3): 249258. 2009.

[11] C. Caicedo and M. Weiss, "The viability of spectrum trading markets," Proceedings of the 2010 IEEE Symposium on New Frontiers in Dynamic Spectrum Access Networks (DySPAN), Singapore: 1-10. 2010.

[12] D. Teece, M. Pisano and A. Shuen, "Dynamic capabilities and strategic management," Strategic Management Journal, Vol. 18 (7), 509-533, 1997.

[13] The White House, "Expanding America's Leadership in Wireless Innovation," Presidential Memorandum, 2013. 
[14] The FCC, "16-55: The Second Report and Order and Order on Reconsideration finalizes rules for innovative Citizens Broadband Radio Service in the $3.5 \mathrm{GHz}$ Band (3550-3700 MHz)," [Online]. Available: https://apps.fcc.gov/edocs_public/attachmatch/FCC-1655A1.pdf., May 2016.

[15] The NTIA, "An Assessment of the Near-Term Viability of Accommodating Wireless Broadband Systems in the 1675-1710 $\mathrm{MHz}, 1755-1780 \mathrm{MHz}, 3500-3650 \mathrm{MHz}$, and 4200-4220 MHz, 4380$4400 \mathrm{MHz}$ Bands," The National Telecommunications and Information Administration, Oct 2010.

[16] F. Abinader et al., "Enabling the Coexistence of LTE and Wi-Fi in Unlicensed Bands," IEEE Communications Magazine 52(11): 54 61. 2014.

[17] M. Sohul, M. Yao, T. Yang and J Reed, "Spectrum access system for the citizen broadband radio service. IEEE Communications Magazine 53(7): 18-25. 2015.

[18] The US Department of Commerce, National Telecommunications and Information Administration (NTIA), "Technical Report 16-521: Using On-Shore Detected Radar Signal Power for Interference Protection of Off-Shore Radar Receivers," May 2016.

[19] The WINNF SSC, "SAS Functional Architecture," [Online]. Available: http://groups.winnforum.org/d/do/8512, Sept 2015.

[20] The Spectrum Sharing Committee of the Wireless Innovation Forum (WINNF SSC), [Online]. Available: http://groups.winnforum.org/page/spectrum-sharing-committee

[21] The WInnF SSC, "WINNF-15-P-0023 Interim SAS to CBSD Protocol Technical Report-A,“ 2015.

[22] The WInnF SSC, "WINNF-15-P-0062-1.0.0 SAS to CBSD Protocol Technical Report-B, “ 2016.

[23] The WInnF SSC, “WINNF-15-P-0051-V1.0.0 SAS to SAS Interface TR-A," 2016.

[24] The WInnF SSC, "WINNF-16-P-0003-1.0.0 SAS to SAS Interface TRB," 2016.

[25] The WInnF SSC, "WINNF-15-S-0112-V1.0.0 CBRS Operational and Functional Requirements," 2016.

[26] P. Aho et al., "Field Trial of Citizens Broadband Radio Service (CBRS). The 22nd European Wireless Conference (EW16), Oulu, Finland. May 2016.

[27] M. Hamblen, "Multiple U.S. trials underway for shared $3.5 \mathrm{GHz}$ wireless spectrum," Computerworld, Inc. [Online]. Available:http://www.computerworld.com/article/3075748/mobilewireless/multiple-u-s-trials-underway-for-shared-3-5ghz-wirelessspectrum.html,May 2016.

[28] The 3GPP Work Item Description, "RP-161219: CBRS 3.5 GHz band for LTE in the United States," The 3rd Generation Partnership Project, June 2016.

[29] The Federal Communications Commission Technical Advisory Council Advanced Sharing and Enabling Wireless Technologies (EWT) WG, "Sharing recommendations," [Online] Available: https://transition.fcc.gov/bureaus/oet/tac/tacdocs/meeting92314/TAC MeetingSummary9-23-14.pdf. Cited 2016/05/19. 2014

[30] The FCC, "16-89 Report and Order and Further Notice of Proposed Rulemaking: Use of Spectrum Bands Above $24 \mathrm{GHz}$ for Mobile Radio Services," July 2016.

[31] C. Wang and P. Ahmed, "Dynamic capabilities: A review and research agenda," International Journal of Management Reviews, Vol. 9 (1), 31 $51,2007$.

[32] S. Zahra, H. Sapienza and P. Davidson, "Entrepreneurship and dynamic capabilities: A review, model and research agenda," The Journal of Management Studies, Vol. 43 (4), 917-955, 2006.
[33] G. Cepeda and D. Vera, "Dynamic capabilities and operational capabilities: A knowledge management perspective," Journal of Business Research, Vol. 60 (5), 426-437, 2007.

[34] C. Prahalad and G. Hamel, "Core competence of the corporation," Harvard Business Review, Vol. 68 (3), 79-91, 1990.

[35] D. Teece, "Dynamic capabilities and strategic management. Oxford University Press, Oxford, 2009.

[36] S. Winter, "Understanding dynamic capabilities. Strategic Management Journal, Vol. 24 (10), 991-995, 2003.

[37] K. Blomqvist and J. Levy, "Collaboration capability - a focal concept in knowledge creation and collaborative innovation in networks," International Journal of Management Concepts and Philosophy, Vol. 2 (1), 31-48, 2006.

[38] T. Eriksson, "Dynamic capability of value net management in technology-based international SMEs," Doctoral dissertation, Turku School of economics, Series A-9:2013, 2013.

[39] P. Ahokangas, M. Matinmikko, L. Minervini, S. Yrjölä, V. Gonçalves and M. Mustonen, "LSA Incentives for incumbent spectrum users in Licensed Shared Access (LSA): A dynamic capabilities view," European Conference on Networks and Communications (EuCNC'2014), June 2014.

[40] 3GPP TR 36.889, "Feasibility Study on Licensed-Assisted Access to Unlicensed Spectrum," 2015.

[41] Qualcomm, "Introducing MuLTEfire: LTE-like performance with Wi-Fi-like simplicity," [Online]. Available: https://www.qualcomm.com/news/onq/2015/06/11/introducingmultefire-lte-performance-wi-fi-simplicity June 2015.

[42] D. Tapscott and A. Tapscott, "Blockchain Revolution: How the Technology Behind Bitcoin Is Changing Money, Business and the World," Portfolio Penguin, May 2016.

[43] G. Boudreau et al., "Interference Coordination and Cancellation for 4G Networks," IEEE Commun. Mag., Apr. 2009, pp. 74-80. IEEE Communications Magazine 47(4): 74 - 81, 2009.

[44] K. Christidis and M. Devetsikiotis, "Blockchains and Smart Contracts for the Internet of Things," IEEE Access, Vol. 4, 2292 - 2303, 2016.

[45] M. Walport, "Distributed ledger technology: beyond block chain," U.K. Government Office for Science, London, U.K., Tech. Rep., Jan. 2016. [Online]. Available: https://www.gov.uk/government/publications/distributed-ledgertechnology-blackett-review

[46] K. Pedersen, Y. Wang, S. Strzyz and F. Frederiksen, "Enhanced intercell interference coordination in co-channel multi-layer LTEadvanced networks," IEEE Wireless Communications, 20(3):120.127, 2013.

[47] S. Yrjola and E. Heikkinen, "Active antenna system enhancement for supporting Licensed Shared Access (LSA) concept, " The 9th International Conference on Cognitive Radio Oriented Wireless Networks and Communications (CrownCom). Oulu, IEEE: 291 - 298. 2014.

[48] The 3GPP, "TR 36.808 Evolved Universal Terrestrial Radio Access (E-UTRA); Carrier Aggregation; Base Station (BS) radio transmission and reception," The 3rd Generation Partnership Project, 2012.

[49] The 3GPP, "TS 36.300, section 22A.1 Stage-2 high level description," The 3rd Generation Partnership Project, 2016. 